\title{
Introduction à une étude préliminaire
}

Marie-Noëlle Polino

\section{OpenEdition}

\section{Journals}

Édition électronique

URL : https://journals.openedition.org/rhcf/681

DOI : 10.4000/rhcf.681

Éditeur

Rails \& histoire

Édition imprimée

Date de publication : 1 mai 2004

Pagination : $9-15$

ISSN : 0996-9403

Référence électronique

Marie-Noëlle Polino, «Introduction à une étude préliminaire », Revue d'histoire des chemins de fer [En ligne], 31 | 2004, mis en ligne le 07 avril 2011, consulté le 22 avril 2022. URL : http:// journals.openedition.org/rhcf/681; DOI : https://doi.org/10.4000/rhcf.681 


\section{Marie-Noëlle POLINO}

\section{Introduction à une étude préliminaire}

Le présent dossier est la première publication relative au programme de collecte d'archives orales engagé par l'AHICF en 2002, au sens où il s'agit de sa première présentation au public, mais aussi de celle de premiers résultats. Une année de «campagne » a eu le double effet de produire une étude de faisabilité, dont le succès permet à la fois cette publication et la poursuite du programme ; mais, aussi, une étude préliminaire qui, dans des conditions qui restaient celles de l'expérience, a construit un matériau définitif, déjà disponible pour la recherche, et donne un avant-goût et de la pratique de la collecte et de ce qui en sera désormais attendu.

\section{Un programme en définition, 2001-2003}

L'ensemble des programmes de collecte heureusement terminés ou en cours, menés par des administrations et des comités d'histoire, qu'ils aient pour cible ou, plutôt, pour « terrain » des corps de l'État, des officiers, des groupes de personnes réunies par des circonstances historiques (combattants, membres de partis, d'équipes ministérielles) avait mis en évidence, pour les responsables de l'AHICF qui en suivaient le déroulement, deux vérités : un programme de collecte ample et rigoureux demande des moyens intellectuels et financiers importants; il ne peut être improvisé et réclame l'implication, pour le moins à moyen terme, de spécialistes formés d'abord à la discipline qui inspire leur questionnement et l'informe par un certain contexte (histoire sociale, politique ; ethnologie, sociologie du travail, des organisations...), ensuite aux techniques de la collecte d'archive orale (ce terme réunissant les aspects technologiques, rhétorique, psychologiques... de l'entretien en tête à tête avec une personne désignée, par elle-même et/ou par le chercheur, comme témoin). Pendant des années, la conclusion fut celle de la prudence : l'AHICF n'était pas - pas encore - à même d'entreprendre avec succès une telle collecte. C'est la richesse en information et la puissance évocatrice des quelques enregistrements récoltés dans le cadre de la préparation du $8^{e}$ colloque de l'association, « Une entreprise 
publique dans la guerre : la SNCF 1939-1945 », redoublée par l'émotion de la parole des témoins exprimée lors de cette réunion, qui a vaincu les résistances.

Le temps employé à la définition du projet paraîtra peut-être long. Il fallait en effet en adapter le rythme aux moyens réduits, au regard de l'ampleur de l'entreprise, dont disposait l'AHICF et qui ont été entièrement mobilisés. Mais, surtout, le projet occupant peu à peu une place essentielle dans les activités de l'association et faisant appel à des concours extérieurs, nous avons choisi d'en envisager tous les aspects de façon critique et réfléchie pour lui assurer, dans la mesure du possible, la durée qui sera la condition de son succès.

De 2001 à 2002, un petit groupe a défini les termes du cahier des charges du futur programme de collecte. Nous remercions vivement pour l'aide qu'ils nous ont apportée à ce stade essentiel madame Agnès Callu, conservateur au Centre historique des Archives nationales et maitre de conférences à l'Institut d'études politiques de Paris, madame Élise Feller, docteur en histoire, chercheur associé au Lasmas (Laboratoire d'analyse secondaire et des méthodes appliquées à la sociologie CNRSEHESS), et monsieur Jean-Pierre Williot, maitre de conférences à l'université de Paris-Sorbonne, qui ont réfléchi avec les membres du Comité scientifique de l'association, en particulier messieurs François Caron, André Blanc, Christian Chevandier, Georges Ribeill, aux conditions et aux orientations d'une collecte d'archives orales dirigée vers la constitution d'un fonds nouveau et vers une histoire orale intéressée d'abord aux témoignages de personnes détentrices d'une expérience professionnelle rare qui, pour diverses raisons, allait rapidement disparaittre avec leur capacité d'en témoigner.

Le « cahier des charges » présenté au conseil d'administration de l'AHICF en 2003 retenait quatre principes.

La collecte d'une «masse critique » d'entretiens qui distinguerait le fonds raisonné ainsi constitué des recherches ponctuelles de sources complémentaires habituellement menées par des chercheurs isolés pour une publication précise, ou, même, des travaux d'histoire orale limités à un groupe restreint, comme celui qu'avait constitué l'enquête «les origines des grandes vitesses ferroviaires en France, une histoire de la décision ", commandée au CNRS et pilotée par l'AHICF de 1990 à 1992 (19 témoins, 19 entretiens), même si les orientations scientifiques adoptées aujourd'hui s'inscrivent dans la continuité de cette démarche. Le résultat à long terme que s'assigne le programme reste la constitution et l'organisation d'un fonds d'archives orales appelé à enrichir le patrimoine national et sa dimension archivistique est essentielle à sa définition et à sa 
conduite. Prévoyant au départ 250 entretiens environ, le programme s'est stabilisé à un total de 150 témoins interrogés, certains plusieurs fois (la durée totale d'enregistrement devant donc atteindre un minimum de 300 heures, un maximum de 500). Par conséquent, un accent particulier devait être mis sur un traitement archivistique complet et réfléchi pour assurer la mise à disposition si possible immédiate des enregistrements : sauvegarde technique ; traitement documentaire. Ces deux aspects sont analysés par Sabine Loupien. Parallèlement, tant pour sauver des fonds d'intérêt général que pour construire le contexte du programme, une enquête devait être menée pour recenser et, si possible, traiter en vue de leur ouverture à la recherche les séries d'entretiens déjà réalisées par le milieu culturel et associatif cheminot dont on sait l'activité et l'attachement à l'histoire des professions.

Pour orienter cette collecte et lui donner un objectif à moyen terme, c'est l'histoire orale qui était privilégiée, avec la décision de constituer parallèlement deux groupes de témoins, l'un des décisions stratégiques intervenues dans le secteur du transport ferroviaire depuis les années 1970, l'autre des évolutions des métiers ferroviaires, de leurs pratiques et perceptions depuis l'après-guerre. L'un et l'autre devaient être menés ensemble pour satisfaire les orientations traditionnelles mais parfois divergentes de l'histoire orale, sinon pour les réconcilier en un fonds cohérent. Plus sérieusement, il s'agissait d'adapter la recherche à la diversité d'un corpus immense en le structurant par ces deux axes et, de fait, leur interaction pendant la première phase de réalisation du projet est à elle seule pleine d'enseignements, comme le montrera la comparaison des modes de travail, d'approche des témoins, de constitution des échantillons et des résultats obtenus lors de l'étude préliminaire exposés ci-dessous par David Lamoureux et par JeanPierre Williot. Enfin, ces deux orientations s'appuyaient sans rupture de continuité sur l'expérience acquise précédemment tant du côté de l'histoire des grandes vitesses (qui préfigure celle, plus générale, de l'ensemble des décisions stratégiques) que de celle des cheminots dans la guerre et l'Occupation, centrée sur leur expérience professionnelle et l'exercice de leurs métiers : même si méthodes, volume, objectifs sont différents, les milieux concernés et leur approche ne sont pas sans parenté.

Par ailleurs, il était envisagé de lier la conduite du programme comme ses résultats progressifs au double contexte de la constitution en cours de fonds d'archives orales et de l'actualité des axes de recherches poursuivis, tant par des réunions entre responsables de collectes que par des colloques, des séminaires, éventuellement des restitutions aux témoins et des débats organisés autour de certains témoignages majeurs 
avec des professionnels en activité en fonction des intérêts actuels des institutions et entreprises susceptibles de contribuer au financement du programme. Publications imprimées et électroniques étaient également prévues. Ce volet du programme n'a pu trouver encore, faute des moyens humains nécessaires, d'application systématique, mais cette livraison de la Revue d'bistoire des chemins de fer en constitue un avant-goût.

Enfin, le cahier des charges établissait le principe d'une « étude de faisabilité » qui serait aussi une première étape, une étude préliminaire du programme. Il fallait mettre à l'épreuve les méthodes de la collecte, vérifier les hypothèses budgétaires, produire surtout un échantillon d'entretiens dont l'évaluation serait décisive pour la suite et l'orientation définitive du programme.

\section{Les questions posées à l'expérience, 2003-2004}

C'est de ces dix-huit mois de travail que rendent compte les trois articles qui suivent.

Ils sont dus à David Lamoureux, docteur en histoire de l'université de Nice - Sophia-Antipolis, qui après avoir soutenu en mars 2004 sa thèse : "Les cheminots du Sud de la France, de la démobilisation au Front populaire, hommes, actions, syndicats ", a pris la direction du premier axe de la collecte, ainsi défini : "L'histoire des métiers [à la $\mathrm{SNCF}$ depuis la Deuxième Guerre mondiale »; à Jean-Pierre Williot, maitre de conférences à l'université de Paris-Sorbonne, responsable du volet « Les choix stratégiques et évolutions des structures de la SNCF, des années 1960 à nos jours » et à Sabine Loupien, qui accompagne le programme depuis 2002 tout en se spécialisant dans le traitement documentaire de l'archive sonore et audiovisuelle par une maitrise de sciences de la documentation et de l'information puis un DESS « conception et gestion des réseaux d'information et de documentation » poursuivis à l'université de Paris viII.

Par rapport au cahier des charges, le programme scientifique de la collecte a été précisé, les procédures d'entretien ont été expérimentées et la nature du traitement archivistique et documentaire a été définie. En voici quelques exemples.

D'abord et surtout, le caractère complémentaire des deux approches a été avéré, ainsi que leur capacité à construire ensemble une vision globale de la SNCF et de son personnel, correspondant au titre général du programme, "La SNCF, 1937-2002. Histoire professionnelle de l'entreprise et de son personnel : corps social, métiers, évolutions et représentations ». 
Du point de vue de la constitution des échantillons, David Lamoureux et Jean-Pierre Williot ont eu dans cette première phase recours aux premiers témoins eux-mêmes pour compléter leurs listes : David Lamoureux s'en explique, le traitement systématique, par exemple par l'informatique, d'une liste de personnes qui serait fournie par la SNCF est impossible, en dehors même de toute limite posée à un tel traitement par le respect de la vie privée, puisque les critères de gestion administrative du personnel d'une entreprise, pendant l'activité ou la période de versement d'une pension, n'ont aucun point de rencontre avec ceux de l'étude qui recherche une conjonction de grade ou fonction / période d'exercice / établissement ou localité d'exercice. En revanche, la consultation, une fois l'interlocuteur déterminé, d'une fiche de carrière reste possible. Il semble qu'il faille s'en remettre davantage que cela n’avait été prévu aux réseaux sociaux existant (voisinage, syndicats, associations). Pour Jean-Pierre Williot, la liste des témoins étant d'abord déterminée par des organigrammes publiés, c'est le contenu de l'entretien qui l'orientera vers les personnes constituant une ressource pour compléter ou éclairer tel ou tel thème. Dans tous les cas, le processus est long qui conduit à la constitution d'un groupe de personnes susceptible de témoigner de la totalité des aspects des évolutions dessinées comme hypothèses et tous ceux, à commencer par les lecteurs de ce dossier, qui pourront contribuer au programme par leurs propos comme par leur documentation personnelle seront assurément les bienvenus.

Un autre enseignement de l'expérience a été, outre les manières les plus appréciées par les différents témoins de prendre contact avec eux et de leur présenter le programme, ainsi que la place qu'ils y occuperont, la façon de les amener à conclure avec l'AHICF le contrat indispensable à l'utilisation des entretiens par la recherche : de ce point de vue, la différence entre les groupes de témoins va jusqu'à l'opposition, la signature d'un écrit décourageant certains de témoigner sur leur métier, alors qu'il libère la parole des acteurs des décisions stratégiques.

Enfin, plusieurs techniques d'enregistrement et de sauvegarde ont été concurremment utilisées et évaluées, le choix principal restant celui d'un enregistrement numérique sur cassette DAT, par la suite gravé sur $\mathrm{CD}$, après, pour les disques de consultation, égalisation du son et analyse thématique matérialisée par des plages. Ces choix sont amenés à évoluer avec la technologie et avec les prescriptions des Archives Nationales en matière d'archives sonores, auxquelles l'AHICF souhaite se conformer.

Bien entendu, une année de collecte et de préservation du patrimoine sonore existant a permis de mesurer les coûts de la collecte et de «tester» plusieurs modes de travail. La structure de l'équipe, le 
mode de fonctionnement général du programme et les échanges entre les différents volets ont été précisés, du moins pour la phase préliminaire : les responsables de la collecte se sont réunis périodiquement, ensemble, avec le secrétariat, et avec des membres du Comité scientifique de l'AHICF, sous la présidence de M. Baufine-Ducrocq, Président Délégué de l'association.

La bonne volonté des partenaires institutionnels de l'AHICF a été sollicitée et nous les remercions très vivement de la bonne grâce avec laquelle ils nous ont répondu mais, surtout, de l'intérêt qu'ils portent au programme. La direction des Archives de France a été pour 2004 le principal contributeur (pour un montant de $15000 \bullet$ ), suivie par la direction de la Recherche et des Affaires scientifiques et techniques du ministère de l'Équipement, des Transports et du Logement, qui a accordé à l'AHICF une subvention de $10000 \bullet$. En effet, si l'AHICF a consacré au programme une part de son fonds associatif, environ 7500 • par an jusqu'à présent, elle ne peut sans mettre en péril la survie de l'ensemble de ses activités lui sacrifier des ressources propres trop importantes et devra subordonner le rythme du programme au renouvellement de ces subventions annuelles, ainsi qu'à celles des entreprises membres de l'Association, en sus de leur cotisation, qui ont atteint en 20047500 • pour la SNCF et 5000 • pour Réseau ferré de France.

\section{Le programme confirmé par l'étude préliminaire : années 2004-2005 et suivantes}

L'approfondissement des orientations thématiques qu'a permis l'étude préliminaire est développé dans les deux articles de David Lamoureux et de Jean-Pierre Williot. Ce dernier insiste sur la singularité de la démarche qu'il a adoptée - une quinzaine d'entretiens destinés à faire participer des témoins majeurs à l'élaboration du questionnement et à bénéficier de leur avis général sur le programme avant de recueillir leur témoignage détaillé - comme sur son bénéfice. Cette première étape conduit naturellement à la deuxième, c'est-à-dire à la première année de «plein exercice » de l'exécution du programme, le postulat de la répartition de la collecte sur plusieurs années étant maintenu - pour autant que les différents concours sollicités en fassent autant. Pendant cette première année, David Lamoureux s'intéressera à trois métiers « disparus », condamnés par l'évolution du système technique ferroviaire mais qui continuent d'être présents dans les représentations tant internes à la société cheminote que dans le grand public (comme les gardesbarrière ou les « caleurs », les enrayeurs qui opéraient sur les triages), tandis que Jean-Pierre Williot, avec le concours de Noëlline Castagnez, 
maître de conférences d'histoire contemporaine à l'IUFM de Versailles, s'attachera à recueillir les témoignages des hauts dirigeants, anciens et actuels, de la SNCF et des anciens titulaires du mandat ministériel des Transports, avant de passer, l'année suivante, à une autre catégorie de personnes.

L'étude préliminaire a donc contribué à la construction du programme autour d'hypothèses qui ont été précisées - la décision, le métier - et d'objectifs scientifiques - l'échantillon, les questions posées. Elle a déterminé son étendue et son rythme, tout en éprouvant son fonctionnement. Surtout, ce qui apparait comme une première étape fructueuse a permis d'asseoir la finalité d'un programme largement appuyé sur des contributions publiques. Leur importance renforce la volonté de l'association de constituer un fonds d'archives orales qui soit, d'une part, d'intérêt national par son volume et par les questions traitées et, d'autre part, immédiatement accessible à la recherche, dans un souci de restitution au public de l'effort de l'État et de transparence des travaux de l'association. C'est pourquoi la diffusion des travaux, les échanges avec les chercheurs susceptibles de profiter de ces sources nouvelles devraient retenir désormais toute notre attention.

Enfin, rien ne serait possible sans ces personnes, que nous désignons commodément mais abusivement par le vocable réducteur de " témoin ", dont la rencontre est toujours une aventure, souvent un plaisir, et qui nous donnent bien davantage que leur expertise et leur expérience professionnelles. Revenons donc à ce point de départ, en conclusion, pour ajouter nos remerciements à ceux que leur expriment les auteurs et souhaiter qu'elles soient très nombreuses à venir contribuer au succès de ce programme. 